\title{
Optimal design of a DC brushless motor for automotive brake actuation
}

\author{
Francesco CAMOZZI ${ }^{1}$, Antonino DI GERLANDO ${ }^{2}$, Massimiliano GOBBI ${ }^{3}$, \\ Giampiero MASTINU ${ }^{3}$, Alessio MIOTTO ${ }^{1}$, Cristiano FISSORE ${ }^{1}$, \\ Paolo RIGHETTINI ${ }^{4}$, Roberto STRADA ${ }^{4}$ \\ ${ }^{1}$ Brembo, Stezzano BG, Italy \\ ${ }^{2}$ Politecnico di Milano, Dep. of Energy, Italy \\ ${ }^{3}$ Politecnico di Milano, Dep. of Mechanical Engineering, Italy \\ ${ }^{4}$ University of Bergamo, Dep. of Engineering and Applied Sciences Italy
}

\begin{abstract}
A Consortium of Companies and Universities cooperated to design and manufacture electric motors for brake actuation. The project, in an Industry 4.0 framework, aimed to combine the design of both product and process.

The electric motors for brakes have been optimized to shrink as much as possible the geometric dimensions while keeping high output torque, with the constraints of reduced production cost and extremely high reliable performance. A smart production plant has been studied and optimized together with the electric motor.

Multi-objective optimization has been used to design the brushless DC motor. Fifteen design variables were considered for the definition of stator and rotor geometry, pole pieces and permanent magnets. The performance indices are peak torque, efficiency, rotor mass and inertia. The design constraints refer to components stress levels and temperature thresholds.

The physical mathematical models used for optimal design refer to electromagnetic field and related currents computation, to thermo-fluid dynamics simulation, to local stress and vibration assessment. A surrogate model based on Artificial Intelligence has been used to speed-up the simulations. An Artificial Neural Network model, trained with an iterative procedure, was employed. Pareto-optimal solutions resulting from the design process are presented. Significant improvements of the performance indices with respect to a reference solution have been found.
\end{abstract}

.Keywords: Electric motor, brake, optimization, Industry 4.0.

\section{Introduction}

The aim of the paper is to present how to construct effectively electric motors for vehicles. The normal automotive paradigm is to conceive both the product and the construction process at the same time. Since we have fully digitized such two activi- 
ties, we also resort to Industry 4.0 paradigm. All the aspects concerning virtual design, virtual production and virtual testing are addressed.

The market penetration of electric vehicles improves in an unpredictable way. KPMG states that "the rate of EV uptake is uncertain, it is unlikely to be slow and linear." [1]. The high purchase cost of EVs is one of the causes that prevents such vehicles to gain a sound penetration in the market.

Our commitment is to produce high quality electric motors at a relatively low cost. This requires mass production, which requires a proper design of the production process, which requires a proper design of the electric motor.

The paper deals with the construction of electric motors for actuating brakes. The original feature of such products is that they are exclusively conceived and manufactured for automotive applications, with safety, lightweight construction and performance as driving issues.

In the literature [2-5], a number of contributions have been given on the design and construction of electric motors for automotive applications. In this paper we will highlight the relevant features of both the design and manufacturing process of electric motors. This contribution seems original.

The paper is organized as follows. At first, the technical specifications and product impact analysis are briefly addressed. Then the design of both the product and the manufacturing process are described. The optimal design of electric motor is addressed. Then, the flexible production line is described.

At the very end, the prototypes are presented.

\section{Product requirements and technical specifications analysis}

Electrically actuated brakes allow brake-by-wire. The advantages of such technology have been described in [6]. Fig.1 shows the reference Brake-by-Wire system for the study, a distributed braking system concept where each actuator includes an electric motor controlled in order to press the brake pads on disks. Both front and rear brake actuators were considered, in order to define the respective sizes of electric motors.

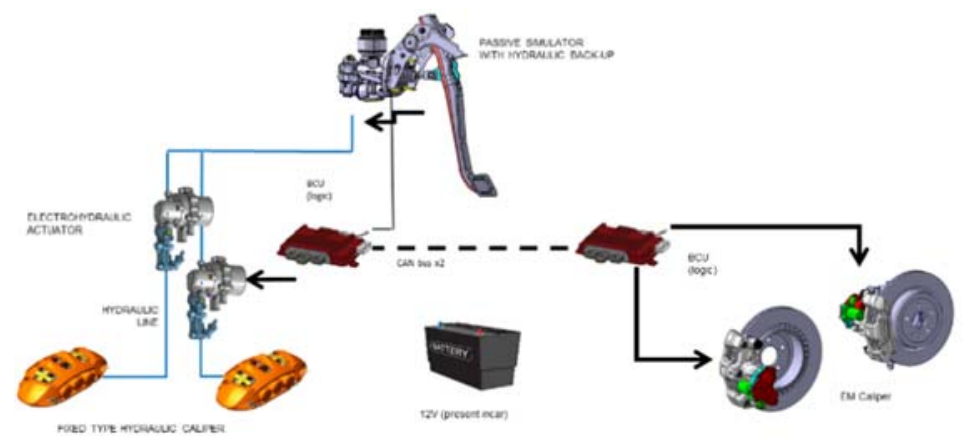

Fig.1. Layout of the Brake-By-Wire system with actuated calipers (Courtesy of Brembo S.p.A). 
In order to address proper design of the electric motors, a system design approach was adopted, starting from the definition of vehicle functions and high-level requirements (including safety aspects). The electric motors requirements were used as input for the optimized motor design.

The front brake actuator motor is shown in Fig. 2. The rear brake actuator is shown in Fig. 3.

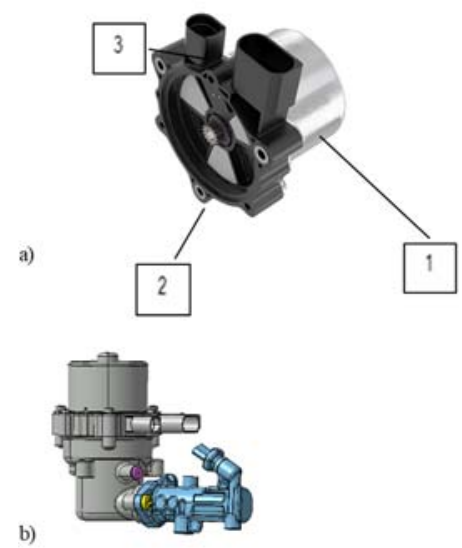

Fig. 2. Front brake hydro-electric motor. a) body of the front brake electric motor. 1: motor body, 2: mechanical interface, 3: electrical interface b) Electro-hydraulic actuator (courtesy of Brembo S.p.a.).

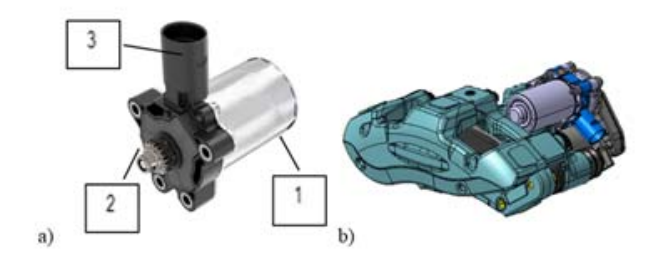

Fig. 3. a) rear brake electric motor. 1: motor body, 2: mechanical interface, 3: electrical interface. b) caliper and electric motor (courtesy of Brembo S.p.a.).

Main design parameters taken into account for the optimal design of the brake motor have been:

- Volume

- Mass

- Max Temp.

- Min Temp 
- Max current

- Nominal Motor Voltage

- No load phase current

- No load speed

- Nominal power

- Max phase current

- Nominal Torque

- Battery Voltage range

\section{Electric motor description (Brushless DC motor - BLDC)}

The brushless DC motors in Figs. 2 and 3 have been optimized. Here the optimization of the motor in Fig.3 is dealt with.

Motor topology (stator and rotor) was selected in order to fit the available room. The rotor magnetic field is generated by surface-mounted permanent magnets (PM) and concentrated stator winding.

The permanent magnets are glued onto the rotor, but bandages are needed to counteract centrifugal forces. A metallic sleeve and a non-metallic bandage (carbon fiber, fiberglass, ....) have been studied.

The number of poles and slots have been set according to known paradigms. The number of poles is an even number, the number of slots is a multiple of the number of phases. The winding factor is maximized. The number of poles is different from the number of slots, to avoid cogging torque. Cogging torque produces unacceptable vibrations.

The material used for the magnets is neodymium iron boron ( $\mathrm{NdFeB})$.

The motor voltage is $12 \mathrm{~V}$. The current input of the 3 phases is defined by the BCU (Brake Control Unit) model that considers the switch and diode characteristics. The actual simulation condition is with constant current value. that allows shorter computational times.

The electric motor is tested at the maximum operative temperature required by the manufacturer, that is $120^{\circ} \mathrm{C}$.

The windings section geometry and placement are defined by using the copper slot fill factor. This factor is kept constant in the optimization process presented in the next sections. The windings pattern through the slots of the stator is fixed for all the motor configurations and equal to the reference design.

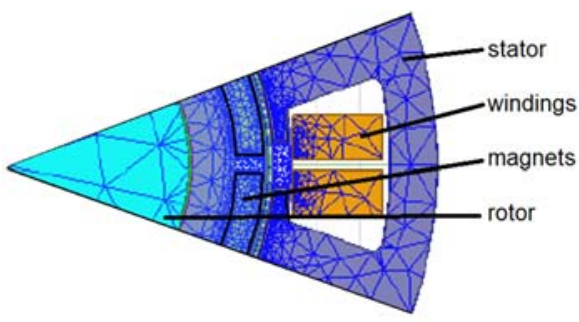

Fig.4. Preliminary design of the BLDC electric motor. 


\section{$4 \quad$ Physical models}

The multi-physics mathematical models of the motor refer to electromagnetic field and related currents computation, to thermo-fluid dynamic simulation, to local stress and vibration assessment (see Fig. 5).

The electromagnetic model is a two-dimensional FE model developed in Motor$C A D \circledR$. The output torque, the cogging torque and the actual power consumption are computed. Operating temperatures are computed by a lumped parameter model. The dependence of magnetic and electrical characteristics is taken into consideration.

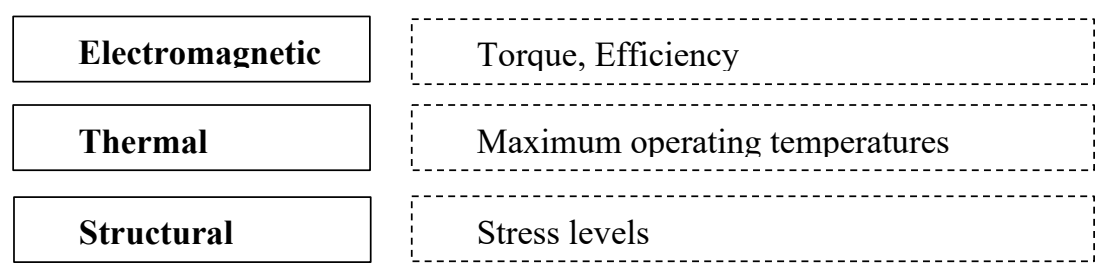

Fig.5. Physical models used in the optimization

A two-dimensional ABAQUS ${ }^{\circledR}$ FE model computes the stress levels. The rotor is the critical part. A Python script is used to update the geometry of the parts for a given set of parameters values and to take into account the mechanical and thermal loading of the components resulting from the Motor-CAD® simulations.

\section{Optimization}

The electric motor optimal design is formulated according to Multi-Objective Programming (MOP).

In a MOP problem, solutions are not unique. Solutions belong to the Paretooptimal set. A preferred solution has to be chosen from this set. Pareto-optimal solutions are the best obtainable compromise among conflicting objectives.

\section{Design variables}

15 design variables pertaining to stator and rotor geometry, pole pieces and permanent magnets are considered. Fig. 5 shows the relevant geometrical design variables.

AutoCAD ${ }^{\circledR}$ is used to parametrize the geometry. An Excel ${ }^{\circledR}$ code is used to update the drawing parameters to feed analysis. A MATLAB ${ }^{\circledR}$ code was developed to import the geometry in Motor-CAD ${ }^{\circ}$. The electromagnetic and thermal analyses are performed as function of the many different geometries. 

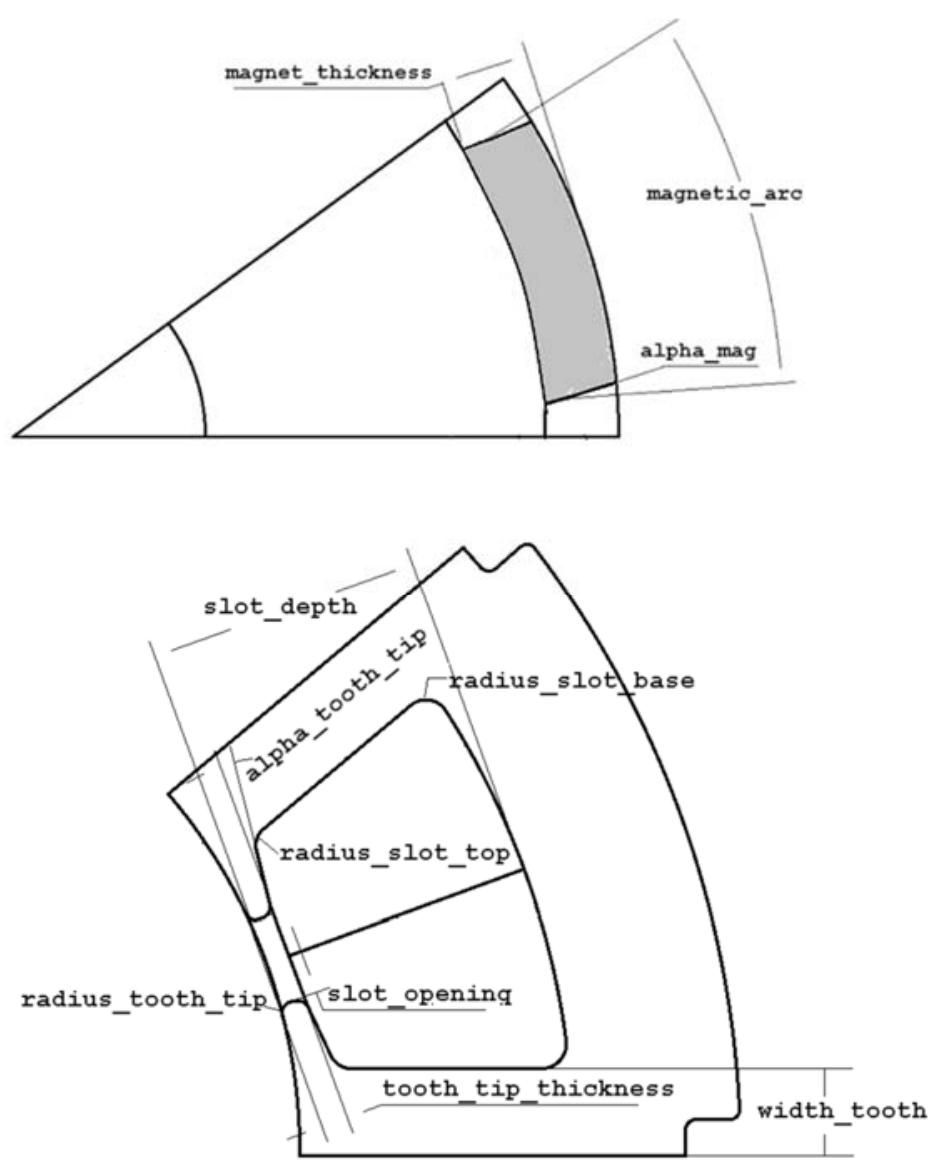

Fig.5. Geometry main design variables, rotor (top), stator (bottom).

\section{$7 \quad$ Objective functions}

The considered three objective functions are

TM maximum holding torque (to be maximised),

Izz rotor inertia (to be minimised)

$\mathrm{P}$ energy consumption while stalling at $1 \mathrm{Nm}$

(efficiency index, to be minimised). 


\section{Constraints functions}

5 geometry constraints have been introduced to avoid non feasible designs (rotor geometry, stator geometry, etc.).

Additionally, design constraints that refer to components stress levels, temperature thresholds and maximum cogging torque are applied.

They read

Twindings $<$ Tw max

Tmagnets $<$ Tmag max

Von Mises, rotor $<\square$ yield

Cogging Torque $<$ Admissible cogging torque

Artificial Intelligence is used reduce the optimization time. One thousand design variable sets are used to simulate the behavior of the electric motor. Physical models (Electromagnetic, Thermal and Structural) are used. The one thousand solutions are used as 'training set'. Deep Learning algorithms (DLA)are used and tested in terms of generalization capability, i.e. the errors of the DLA have been computed on a 'validation set' constituted by 100 designs not belonging to the 'training set'. If the accuracy is not adequate, the process can be iterated by increasing the number of simulations used to train the DLA and/or by varying the DLA architecture.

The DAL is thousands of times faster than the physical models used to train it (120s per design for the physical model, $0.01 \mathrm{~s}$ for the DAL).

The trained DLA has been used to evaluate the performances of a large set of experiments (1 million different design solutions).

Further details on optimization are provided in [12]

\section{Design of Experiments}

When the dimensionality of the problem increases, the design variables domain volume increases so fast that the available data become sparse and the amount of data needed in the sampling phase grows exponentially with the dimensionality (curse of dimensionality). Several sampling techniques [12] can be employed in order to define "low discrepancy sequences" having almost the same discrepancy of a uniform grid but using a limited number of samples. 


\section{Deep Learning (Artificial Intelligence)}

The DAL architecture is based on Artificial Neural Networks (ANN). A multilayer neural network was used [12]. A feedforward backpropagating neural network (ANN) with 15 inputs, 6 outputs and 2 hidden layers has been designed [12].

\section{Search Method}

The Parameter Space Investigation (PSI) method has been applied [12]. The feasible and Pareto optimal solutions are determined by applying the definition of Paretoset. More details in [12].

\section{Optimization results}

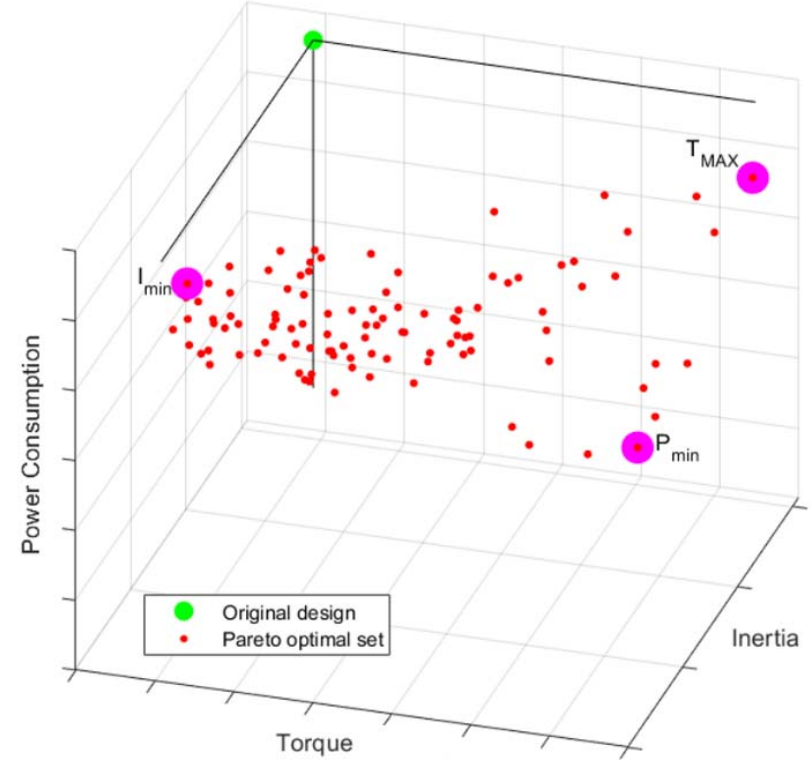

Fig. 9. Pareto Optimal solutions.

A preliminary design provided an electric motor with a very good performance, however the optimization allowed even better behavior. 


\subsection{Max torque solution}

The torque at low speeds is the most relevant index of performance.

All the design constraints (cogging torque, maximum stress levels, maximum operative temperature) are satisfied.

Referring to the design variables, the stator section has been widened especially at the tooth tip and stem region. This leads to a reduction in the magnetic saturation of the ferromagnetic material that constitutes the magnetic circuit, allowing for a larger magnetic flux value and thus more torque. The curvature radii at the tooth tip and slot corners are reduced, but are within the feasibility constraints. More details in [12].

\subsection{Min Inertia}

The rotor inertia is reduced by almost $35 \%$. This is achieved by a $12 \%$ reduction of the stator bore radius. The torque characteristic is very close to the original one across all the speed ranges and the power consumption very close to the original one.

\section{D-FMEA process}

The brake electric motors that have been developed are safety components, so a comprehensive Design FMEA has been carried out from the initial stage of the development, in synergy with the buildup of the Process FMEA.

\section{Flexible line design}

One of the main innovative characteristics of the project is the integrated design of product and process taking into account the effects that each product specification may have on the relevant phase of the manufacturing process. Since the flexibility of the production line is paramount, the opportunities that its complete digitalization offers have been used to achieve the following targets [11]:

1. The processing of a batch of products also with the possibility of a production schedule customizable according to the needs;

2. The processing of a single B-PMM or even only one of its subassemblies with the same smart and flexible approach of the previous mode.

What has been stated introduces an innovative work method that enables the line to be adequate with all the different phases of a product development, in particular with the phases of:

1. Prototyping;

2. Series production; 
Focusing on the first one, the prototyping requires the management of a variety of parameters and, for each of them, of different values. For this reason, the flexibility has been also designed to satisfy the maximum range of values that may be used during the testing activities. Moreover, for the balancing and sizing of the flexible line, also several discrete - event simulations have been carried-on and a wide range of ergonomic and performance indexes have been taken into consideration [12].

\subsection{Process steps and layout design}

The integration of design and manufacturing development has allowed to analyze the production technology in order to scale the machine performance and optimize the B-PMM components design "Design for manufacturing" way.

According to what has been previously mentioned and since the line produces a variety of B-PMM, the process of manufacturing has been designed to work in parallel with the following main subassemblies:

1. Stator;

2. Rotor;

3. Flange;

4. Motor.

While considering the requirements of production of each component, the layout design has been developed in order to achieve an accurate space-saving, reducing the surface needed and improving the process capability.

The flow chart is shown in the following Fig. 10 in which the main subassemblies have been reported.

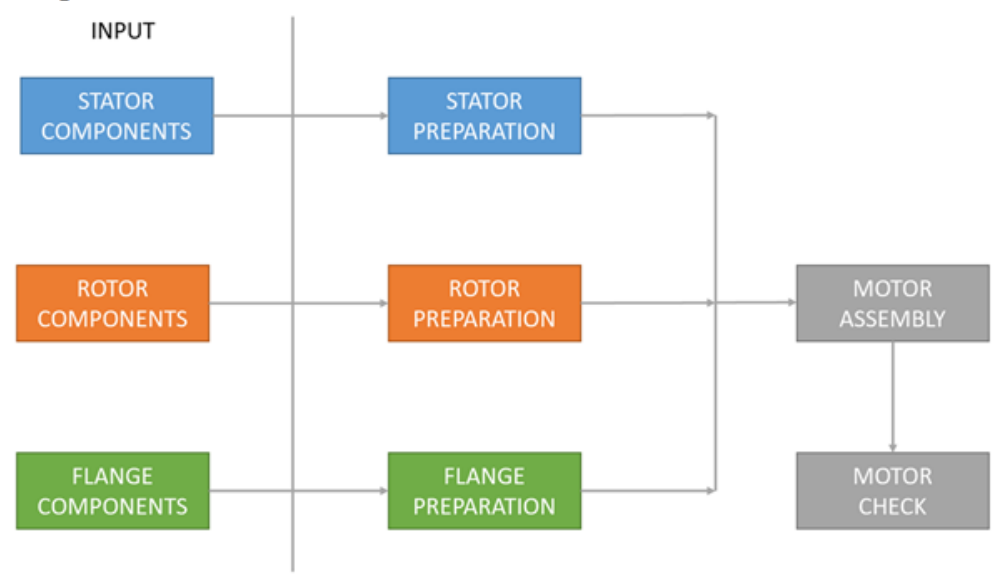

Fig. 10: Production flow chart 


\section{PFMEA of B-PMM products}

Since the B-PMM requires a strict compliance with the requirements about safety of the vehicle, a scientific and quantitative approach has been introduced and applied during all the steps of the project in order to guarantee each phase of manufacturing to work according to what has been declared [11]. For these reasons, the predictive approach known as FMEA, acronym of Failure Mode and Effects Analysis, has been selected to evaluate the effectiveness of the choices stated on the real case of manufacturing, and for this reason called FMEA of Process (PFMEA).

More specifically, the PFMEA tool has been largely applied to check and avoid the failures that may occur during the production [9][10].

An integration of FMEA's, Design and Process, allows to align the functional requirements of B-PMM from the design to the manufacturing, in order to get, through such synergic approach, the best performance of the product and the quality level required [9][10].

The output of this analysis is a unique parameter (Risk Priority Number RPN) useful to check the functions; if they have this value higher than a stated limit of acceptance, it means that they need an improvement effort.

An example of the relationships existing between causes and failures is provided in Fig.11. In the same figure, the different levels above mentioned are shown [11].

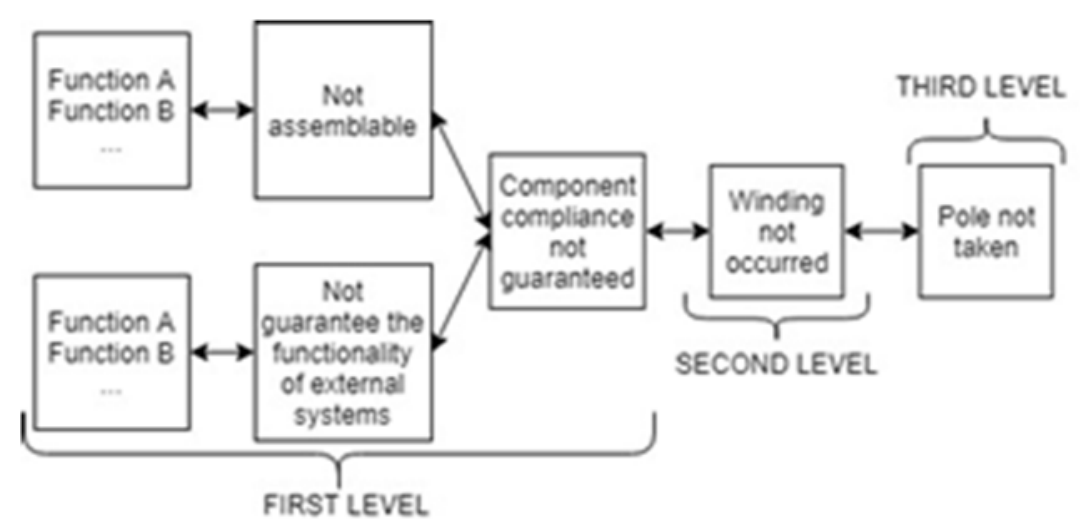

Fig. 11. Example of the connections between failures

\subsection{Production process checks and quality design}

As above mentioned, in order to comply with the requirements, the product must be checked during the process of manufacturing.

The design of each manufacturing step is made with the Total Quality Management approach. 
Based on PFMEA, causes and effects failures analysis, the controls are tailored on the functional characteristics of process and design.

Related to the most critical phases of work, checks based on different technologies are performed, with the final scope to monitor the process and to store information.

All manufacturing steps are monitored by means of one or more parameters. The reason for each control is to evaluate the manufacturing process and to monitor the process steps in order to prevent production derivation and scrap generation; moreover, such controls allow to guarantee the functional requirement stated during the design phase. A positive result of this control enables the component to proceed according to its flow chart, otherwise it may be considered to be reworked or, at least, a waste.

Furthermore, the phase of quality control of the B-PMM is the final step of the process of manufacturing and its target is to verify the compliance with its main specifications. According to this, the specifications must be pointed out as measurements needed to be performed by the workstation of motor check and guaranteed through an adequate phase of design for the integration of the different measurement systems.

As a result, the output of the "production report" is a detailed report that contains all the information picked up by sensors and the final statement about quality and history of each B-PMM produced on the line.

\subsection{IT architecture $\backslash$ and IoT integration}

Since the principles of the 4.0 Industry have been introduced, the traceability of each single item in term of part number as well as of the working phases must be guaranteed. In order to allow this, a complete digitalization of the entire process of manufacturing through an adequate structure of data management has been designed.

In this instance, the production is monitored by a Supervisor System Unit (SUU), linked to the stations by a specific logic and architecture. The communication of all data is done by standard protocol applied for each machine.

The data acquired by sensors are managed by the SSU that can act on the parameters achieving the results while preventing risks and avoiding damages [11].

This implies that the SSU must be provided with an integrated knowledge about the variety of products and recipes available, that implies different data acquired that must be stored in different formats in order to enable the next phases of Data Analysis.

As a result, the SSU allows, on one hand, the control of the real time production pointing out the actual state of completion of the product and the relevant performance achieved by the line, and, on the other, provides the data for the detailed historical report of the B-PMM already made. 


\section{Conclusions}

Two electric motors, fully engineered with their own production lines, have been presented. The two motors allow brake-by-wire and are devoted to the front and rear wheels of a reference car, respectively. All of the electric motors have been designed and manufactured after an in-depth optimization process. Such a process referred to both product design and production line engineering. The electric motors will contribute to the penetration of electric vehicles into the market.

\section{References}

1. KPMG, https://assets.kpmg/content/dam/kpmg/au/pdf/2018/electric-vehicles-is-theenergy-sector-ready.pdf

2. Momen, F., Rahman, K., Son, Y., and Savagian, P., "Electric Motor Design of General Motors' Chevrolet Bolt Electric Vehicle," SAE Int. J. Alt. Power. 5(2):286-293, 2016, https://doi.org/10.4271/2016-01-1228.

3. A Giorgetti, L Cavestro, M Rampazzo, Design of a Lightweight Braking System for Electric Cars, SAE Technical Paper 920649 doi: https://doi.org/10.4271/920649

4. Ramakrishnan, K., Romanazzi, P., Zarko, D., Mastinu, G. et al., "Improved Analytical Model of an Outer Rotor Surface Permanent Magnet Machine for Efficiency Calculation with Thermal Effect," SAE Int. J. Alt. Power. 6(1):34-46, 2017, https://doi.org/10.4271/2017-01-0185.

5. A Carriero, M Locatelli, K Ramakrishnan, G Mastinu, and M Gobbi, A Review of the State of the Art of Electric Traction Motors Cooling Techniques, SAE Technical Paper2018-01-0057 ISSN: 0148-7191, e-ISSN: 2688-3627, doi: https://doi.org /10.4271/2018-01-0057

6. Sundar, M. and Plunkett, D., "Brake-by-Wire, Motivation and Engineering - GM Sequel," SAE Technical Paper 2006-01-3194, 2006, https://doi.org/10.4271/2006-01-3194.

7. Mastinu, G. Ploechl, M, Road and off-road Vehicle System Dynamics Handbook, CRC Press, Boca Raton, New York, USA, 2014

8. Mastinu, G., Gobbi, M., Miano, C, Optimal Design of Complex Mechanical Systems, Springer, Berlin 2006

9. "Potential Failure Mode and Effects Analysis (FMEA) - Reference Manual Fourth Editon", AIAG (Automotive Industry Action Group), April 1993.

10. "SAE AS13004 - Process Failure Mode and Effects Analysis (PFMEA) and Control Plans", 2017.

11. Righettini, P., Strada, R., Gosatti, A., Togni, S., Camozzi, F., Fissore, C., "Smart Mobility: a modern approach to automotive product development for vehicle electrification", Proceedings of IEEE RTSI 2019 (5th International Forum on Research and Technologies for Society and Industry), Firenze, 9-12 september 2019.

12. Righettini, P., Strada, R., Togni, S., Cortinovis, F., Fissore, C., "Sizing and Balancing of a Semi-Automated Line for Automotive Electric Motors by Means of Ergonomic And Performance Analysis", International Journal of Engineering and Advanced Technology (IJEAT). 9(4): 2305-2317, 2020. doi:10.35940/ijeat.D9079.049420

13. Di Gerlando, A., Gobbi, M., Mastinu, G., and Miotto, A., "Electric Motor for Brakes - Optimal Design," SAE Technical Paper 2020-01-0919, 2020, doi:10.4271/2020-01-0919. 